\title{
Theileria orientalis Ikeda Genotype in Cattle, Virginia, USA
}

\author{
Vanessa J. Oakes, Michael J. Yabsley, Diana Schwartz, Tanya LeRoith, \\ Carolynn Bissett, Charles Broaddus, Jack L. Schlater, S. Michelle Todd, \\ Katie M. Boes, Meghan Brookhart, Kevin K. Lahmers
}

Theileria orientalis Ikeda genotype is a parasite that causes a disease in cattle that results in major economic issues in Asia, New Zealand, and Australia. The parasite is transmitted by Haemaphysalis longicornis ticks, which have recently been reported in numerous states throughout the eastern United States. Concurrently, cattle in Virginia showed clinical signs consistent with a hemoprotozoan infection. We used amplicons specific for the major piroplasm surface protein and small subunit rDNA of piroplasms to test blood samples from the cattle by PCR. Bidirectional Sanger sequencing showed sequences with $100 \%$ identity with T. orientalis Ikeda genotype 2 sequences. We detected the parasite in 3 unrelated herds and from various animals sampled at 2 time points. Although other benign $T$. orientalis genotypes are endemic to the United States, detection of $T$. orientalis Ikeda genotype might represent a risk for the cattle industry in Virginia.

$T$ heileria orientalis is an emerging parasitic pathogen of cattle that was originally identified in the Eastern Hemisphere (1). The taxonomy of this group is evolving (2-4); taxonomic classification relies on sequencing of 2 major genes: the small ribosomal subunit (SSU) and the major piroplasm surface protein (MPSP). Use of the names of $T$. orientalis genotypes Ikeda, Chitose, and Buffeli is embedded in the clinical literature; these designations are used throughout this article. A genotype scheme proposed in 2014 classifies $T$. orientalis into 11 genotypes according to variability in the MPSP gene (4); T. orientalis genotype Ikeda correlates with genotype $2(3,5)$.

Consistent with other members of the genus, T. orientalis is a tickborne hemoprotozoan with a life cycle

\footnotetext{
Author affiliations: Virginia-Maryland College of Veterinary Medicine, Blacksburg, Virginia, USA (V.J. Oakes, T. LeRoith, S.M. Todd, K.M. Boes, M. Brookhart, K.K. Lahmers); University of Georgia, Athens, Georgia, USA (M.J. Yabsley); Kansas State University, Manhattan, Kansas, USA (D. Schwartz); Virginia Department of Agriculture and Consumer Services, Richmond, Virginia, USA (C. Bissett, C. Broaddus); US Department of Agriculture, Ames, lowa, USA (J.L. Schlater)
}

DOI: https://doi.org/10.3201/eid2509.190088 that affects erythrocytes and leukocytes and contributes to chronic anemia, ill-thrift, and persistent subclinical infections. However, it has not been associated with the lymphoproliferative disease seen with T. parva and T. annulata (1). Haemaphysalis spp. ticks are the primary biological vector of $T$. orientalis ( 6 ) and are believed to be essential for completion of the $T$. orientalis life cycle (7), although there is limited evidence suggesting that transmission might occur through flies, lice, or vaccine needles (1).

In Asia, New Zealand, and Australia, theileriosis caused by $T$. orientalis is an economically serious disease manifested primarily by loss of revenue from deaths or illness in beef and dairy cattle $(1,8-10)$. Of increasing concern is the Ikeda genotype of $T$. orientalis, which has been implicated as the etiologic agent of infectious bovine anemia $(11,12)$. In Asia, Australia, and New Zealand, the primary tick vector for the $T$. orientalis Ikeda genotype is Haemaphysalis longicornis, which is also known as the Asian longhorned or bush tick.

The Asian longhorned tick was first detected in the United States in August 2017 and has subsequently been detected in New Jersey (13), New York, North Carolina, Virginia, West Virginia, Pennsylvania, Maryland, Connecticut, and Arkansas $(14,15)$. However, examination of archived tick samples has identified $H$. longicornis ticks in the United States since 2010 (14). Because of the wide host range of this tick, its bisexual nature, and its ability to reproduce parthenogenetically (16), concern is increasing that there are established populations in the mid-Atlantic states.

In September 2017, a beef cattle herd in Virginia was given a diagnosis of anemia and suspected anaplasmosis. Blood samples were negative for Anaplasma marginale ticks by PCR, but blood smears showed numerous pleomorphic piroplasms. We report identification and characterization of $T$. orientalis Ikeda genotype 2 from an index farm, in adjacent herds, and 2 other counties in Virginia. Although other genotypes of $T$. orientalis are present in the United States (17-19), the Ikeda genotype in particular has not been identified in North America and represents an emerging infectious disease with potential for major animal 
health and economic impacts, especially because a competent vector has been identified in this region and in 2 of the 3 farms described in this report.

\section{Materials and Methods}

\section{Animals}

All cattle were client-owned animals. In August 2017, seven cattle from a herd in Albemarle County, Virginia, died after showing adverse clinical signs, including weakness and malaise. Affected cattle included bulls, cows, and steers ranging in age from 3 months to 13 years. All animals were born and raised on a farm. In September 2017, an additional cow from the index farm was examined for weakness, icterus, and anemia (packed cell volume [PCV] $12.0 \%$ ). Blood from this animal was collected and submitted to the Kansas State Veterinary Diagnostic Laboratory (Manhattan, KS, USA). Blood smear analysis showed evidence of a hemoprotozoal infection (Figure 1). Molecular testing of this sample resulted in a diagnosis of infection with $T$. orientalis, which prompted quarantine of the affected farm and further investigation.

A foreign animal disease investigation was instituted during December 2017, and blood was collected from the index cow and 5 additional, randomly sampled cattle from the herd. We collected blood by jugular vein venipuncture from each animal in 10-mL BD Vacutainer plastic red-top tubes containing no anticoagulant and in $10-\mathrm{mL}$ BD Vacutainer plastic purple-top tubes containing EDTA

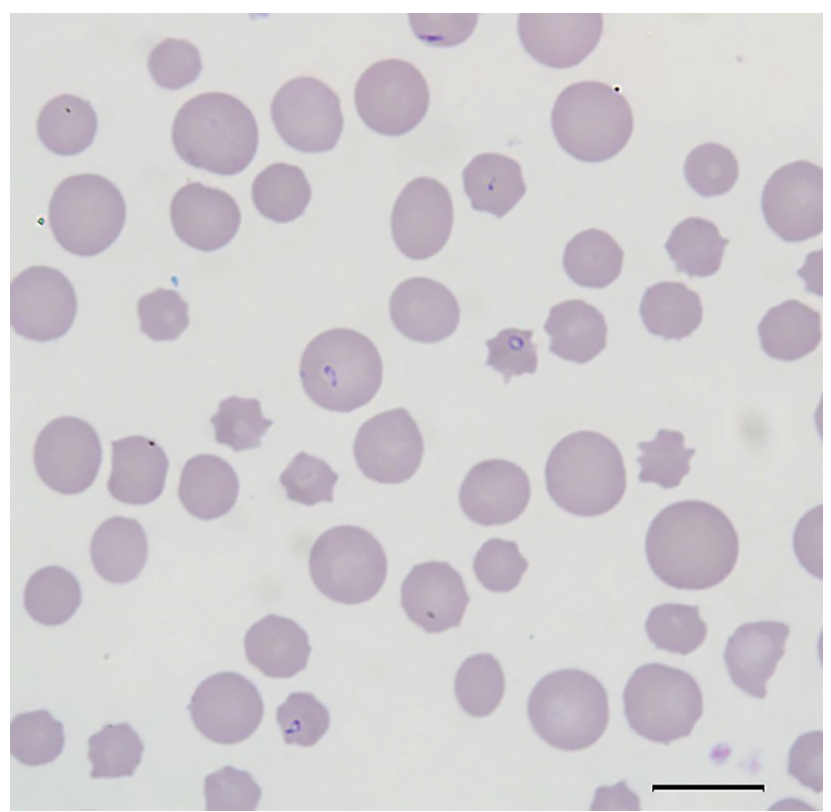

Figure 1. Blood smear of an animal from a farm in Albemarle County, Virginia, USA, that was infected with Theileria orientalis Ikeda genotype. There is evidence of a regenerative response to anemia (anisocytosis and polychromasia) and intracellular piroplasms within erythrocytes. Scale bar indicates $10 \mu \mathrm{m}$. anticoagulant (both from Becton, Dickinson and Company, https://www.bd.com) as part of a routine diagnostic investigation for suspected anaplasmosis or as part of a random sampling effort.

\section{DNA Extraction and PCR Testing}

We extracted DNA from EDTA anticoagulant blood by using the DNeasy Blood and Tissue Kit (QIAGEN, https:// www.qiagen.com) according to the nonnucleated blood protocol. We performed the final elution stage by using 50 $\mu \mathrm{L}$ of nuclease-free water and incubating the spin column membrane for $1 \mathrm{~min}$ at room temperature. This step was repeated to yield a final elution volume of $100 \mathrm{~L}$.

We performed amplification for the MPSP by using 10.5 $\mu \mathrm{L}$ of DNA in a $25-\mu \mathrm{L}$ reaction volume containing primers MPSP forward (5'-CTTTGCCTAGGATACTTCCT-3') and MPSP reverse (5'-ACGGCAAGTGGTGAGAACT-3') as described $(11,20)$. Each amplification had a final reaction primer concentration of $0.4 \mu \mathrm{mol} / \mathrm{L}$.

We performed amplification for the internal segment of the SSU by using $10 \mu \mathrm{L}$ of DNA in a $25-\mu \mathrm{L}$ reaction volume containing primers SSU internal forward (5'-ATTGGAGGGCAAGTCTGGTG-3') and SSU internal reverse (5'-CTCTCGGCCAAGGATAAACTCG-3') as described $(11,20)$ and identical PCR protocols as we described previously. We also used a Biometra TProfessional Thermocycler (AnalytikJena AG, https://www.analytik-jena.com).

We visualized amplicons by electrophoresis on a $1.0 \%$ agarose gel containing ethidium bromide and Tris-borateEDTA buffer and examined amplicons by using UV transillumination. Amplicons with sizes of 700-800 bp were submitted to the Virginia Biocomplexity Institute (Blacksburg, VA, USA) for bidirectional Sanger sequencing.

\section{Anaplasmosis Testing}

Animals sampled as part of a foreign animal disease investigation were tested for Anaplasma, Babesia, and Leptospira spp. at the National Veterinary Services Laboratories (NVSL; Ames, IA, USA). The other animals involved in this study were tested for Anaplasma marginale by using a quantitative PCR used and validated by the Virginia Tech Animal Laboratory Services (ViTALS, Blacksburg, VA, USA) diagnostic laboratory (21) (Table).

For the samples tested by ViTALS, we extracted DNA from blood by using the protocol we described previously. DNA samples were diluted 1:10 with nuclease-free water. Amplifications were performed by using AM-For $16 \mathrm{~S}$ forward primer (5'-TTGGCAAGGCAGCAGCTT-3') and AM-Rev 16S reverse primer (5'-TTCCGCGAGCATGTGCAT- $\left.3^{\prime}\right)$ at a concentration of $0.6 \mu \mathrm{mol} / \mathrm{L}$ each, and $\mathrm{AM}-\mathrm{Pb}$ probe (5'-6-FAM/TCGGTCTAACATCTCCAGGCTTTC AT/3BHQ_1-3') at a concentration of $0.2 \mu \mathrm{mol} / \mathrm{L}$. Reactions were performed in an ABI 7500 Fast thermocycler 
Table. Diagnostic testing results for Theileria orientalis Ikeda genotype in cattle, Virginia, USA*

\begin{tabular}{lcccc}
\hline Animal ID & Icterus/PCV/parasitemia & Anaplasma tick & Origin & Date \\
\hline I1 & Yes $/ 12.0 \% / 6 \%$ & Negative (NVSL) & Albemarle (Index farm) & Sep 2017, Dec 2017 \\
I2 & No & Negative (NVSL) & Albemarle (Index farm) & Dec 2017, May 2018 \\
I3 & No & Negative (NVSL) & Albemarle (Index farm) & Dec 2017, May 2018 \\
I4 & No & Negative (ViTALS) & Albemarle (Index farm) & Jul 2018 \\
I5 & No & Negative (ViTALS) & Albemarle (Index farm) & Jul 2018 \\
Al1 & No & Negative (ViTALS) & Albemarle & Aug 2018 \\
Al2 & Yes/10.0\%/NA & Negative (ViTALS) & Albemarle & 2018 \\
P1 & Yes/14.4\%/16.4\% & Positive (ViTALS) & Pulaski & Oct 2018 \\
A1 & No & Negative (ViTALS) & Oct 2018 \\
A2 & No & Negative (ViTALS) & Oct 2018 \\
\hline *ID, identification; PCV, packed cell volume; NA, not available; NVSL, National Veterinary Services Laboratories; ViTALS, Virginia Tech Animal \\
Laboratory Services.
\end{tabular}

(Thermo Fisher Scientific, https://www.thermofisher.com) as described (21).

\section{Sequence Analysis}

We examined Sanger sequences of SSU and MPSP amplicons for quality and integrity and generated a consensus sequence by using Geneious Prime R11 (Biomatters, https://www.geneious.com). For paired samples of insufficient quality to form a consensus sequence, the highest quality sequence of the 2 sequences was used. We then aligned consensus sequence extractions with sequences of $3 \mathrm{~T}$. orientalis Ikeda genotypes (GenBank accession nos. AB581627, AP011946, and D11046) by using Geneious Prime.

\section{Phylogenetic Analysis}

To examine the phylogenetic relationship of the cattle parasite in Virginia with other Theileria species, including the 3 genotypes of $T$. orientalis, we constructed a neighbor-joining tree by using a Tamura-Nei genetic distance model with 100 replications. We used the phylogenetic tree for the MPSP gene to best illustrate the relatedness of the samples from cattle in Virginia to the described T. orientalis genotypes (4). Phylogenetic analysis for the SSU gene included the $T$. orientalis Chitose genotype (GenBank accession no. AB520954), 2 T. orientalis Buffeli genotypes (accession nos. AB520955-6), 2 T. orientalis Ikeda genotypes (accession nos. AB520957-8), and $T$. annulata (accession no. AY524666) and T. parva (accession no. AF013418) as outgroups.

\section{Results}

\section{Animals}

Serologic analysis performed at NVSL for the 6 cattle tested showed negative results for Anaplasma, Babesia, and Leptospira species; all 6 animals were infected with piroplasmid hemoparasites on the basis of blood smear review. Subsequent blood samples were collected during May 2018 from 7 animals within the index herd. Two of these animals previously had intracellular piroplasms; 5 of these animals were sampled randomly. Multiple cattle were infested with ticks, which were morphologically identified as $H$. longicornis by NVSL (14). In July 2018, blood samples were collected from 21 cattle representing animals from the index herd and others owned by the same producer on other farms in the county. Before the day of collection, there had been no contact among cattle from different herds. The 21 samples collected represented blood from 20 previously unsampled animals (sampled randomly) and 1 from a calf that had been piroplasm positive during May 2018 .

A random sample from a livestock auction from a cow in Albemarle County was submitted during August 2018 (Al1). Four additional samples were submitted from animals in Augusta (A1, A2), Pulaski (P1), and Albemarle (A12) Counties during October 2018. These animals were unrelated to the cattle at the index farm and had no contact with each other. Of these 5 samples, 3 samples were random samples from a livestock auction (A1, A2, A11), and 2 samples were submitted for evaluation of clinical disease (P1, A12). All animals, except P1, were negative for Anaplasma marginale. On the basis of a blood smear, P1 had $16 \%$ of its erythrocytes parasitized by piroplasms (Table). In addition, this animal had a marked macrocytic, hypochromic, regenerative anemia, hyperbilirubinemia, and icteric plasma consistent with hemolytic anemia (PCV 14.4\% [reference range $24.0 \%-46.0 \%$ ]; mean corpuscular volume $94.4 \mathrm{fL}$ [reference range $40.0 \mathrm{fL}-60.0 \mathrm{fL}$ ]; mean corpuscular hemoglobin concentration $27.3 \mathrm{~g} / \mathrm{dL}$ [reference range $30.0 \mathrm{~g} / \mathrm{dL}-36.0 \mathrm{~g} / \mathrm{dL}$ ]; reticulocytes $194,400 / \mu \mathrm{L}$ [reference range not established]; and total bilirubin $4.1 \mathrm{mg} / \mathrm{dL}$ [reference range $0.1 \mathrm{mg} / \mathrm{dL}-0.6 \mathrm{mg} / \mathrm{dL}]$ ). Blood smear analysis revealed a $16 \%$ parasitemia. P1 died shortly after the blood draw; another animal on the farm had icterus and died 3 days earlier. Al2 had a PCV of $10.0 \%$ and showed signs of icterus and lethargy.

\section{Blood Smears}

The blood smear examinations for the cow that became ill during September 2017 showed evidence of a regenerative response to anemia (anisocytosis, polychromasia, basophilic stippling) and numerous oval, racquet, signet ring, and 
linear piroplasms within erythrocytes (Figure 1). During May 2018, blood smear analysis on 7 additional, randomly sampled animals did not show piroplasms. Two of these samples were from animals that had blood collected and analyzed by NVSL during December 2017; both of these animals were positive for piroplasms in blood smears at that time.

\section{PCR}

Molecular testing of the initial blood sample from the cow that became ill during September 2017 (I1) resulted in a diagnosis of infection with $T$. orientalis. This finding, which, in conjunction with the severity of the clinical signs in this animal and death of previous animals on the premises, led to further investigation of the genotype of the organism and quarantine of the affected farm.

Five of the 6 blood samples taken from cattle during December 2017 were positive by MPSP or SSU assays. Six of 7 cattle from the index herd sampled during May 2018 were positive by MPSP and SSU assays. Of the additional 21 samples collected during July 2018 from the index herd and adjacent properties, only samples from 3 animals were negative by MPSP and SSU assays. Three cattle from Augusta and Pulaski Counties (A1, A2, P1) and 2 cattle from an unrelated herd in Albemarle County (A11, A12) were positive by MPSP and SSU assays. A total of 34 sequences were available for analysis, representing 31 unique animals from multiple farms across southwestern Virginia.

Two cattle (I2 and I3) were positive by MPSP and SSU assays during December 2017, and remained positive by these assays during May 2018. One animal was positive by MPSP and SSU assays during May 2018, and remained positive for both assays during July 2018 . The presence of positive samples at a sampling interval of 1-5 months suggests a chronic or persistent component of the infection.

\section{Sequence and Phylogenetic Analysis}

All 34 MPSP gene (719 bp) sequences from cattle from multiple farms in all 3 counties in Virginia sampled were identical to each other and to $T$. orientalis Ikeda and genotype 2 sequences from GenBank (Figure 2). MPSP sequences of animals I2 and I3 sampled during December 2017 and May 2018 were identical.

On the basis of MPSP gene sequence phylogeny reported by Sivakumar et al. (4), all clinical samples clustered together, along with $T$. orientalis Ikeda and genotype 2 sequences retrieved from GenBank (Figure 3). The next closest related branch was composed of the cluster of $T$. orientalis genotype 7 . Outgroups composed of $T$. annulata and T. parva clustered appropriately. On the basis of SSU gene sequence phylogeny $(4,22)$ all clinical samples clustered together, along with $T$. orientalis Ikeda genotype sequences from GenBank (Figure 4).

\section{Discussion}

Genotypes of Theileria orientalis are native to the United States, but are of the Buffeli genotype and are typically nonpathogenic (17-19). T. orientalis Ikeda/genotype 2, a novel, virulent genotype, has not been previously identified in North America. Although the vector of $T$. orientalis among cattle in Virginia is unknown, H. longicornis ticks are a major vector in New Zealand, Australia, and Asia (1). The recent identification of this tick in the United States and clinical signs of anemia in an Anaplasma-negative herd of cattle infested by $H$. longicornis ticks prompted further investigation into the genotype of $T$. orientalis detected in the cattle sampled.

MPSP is an antigenic marker of Theileria spp., and is used to genotype the 11 Theileria groups $(4,5)$. The sequences analyzed in this study are phylogenetically consistent with genotype 2, equivalent to the Ikeda genotype. Because the samples examined in this study represent individual cattle from geographically distant herds, there is concern that $T$. orientalis Ikeda/genotype 2 could be widespread in the region. This concern is especially potent given the presence of a known vector, $H$. longicornis ticks, within the region simultaneously (14). No ticks from the region have been tested for $T$. orientalis. Thus, further work is needed to better understand transmission of $T$. orientalis Ikeda by $H$. longicornis ticks or other tick species in Virginia.

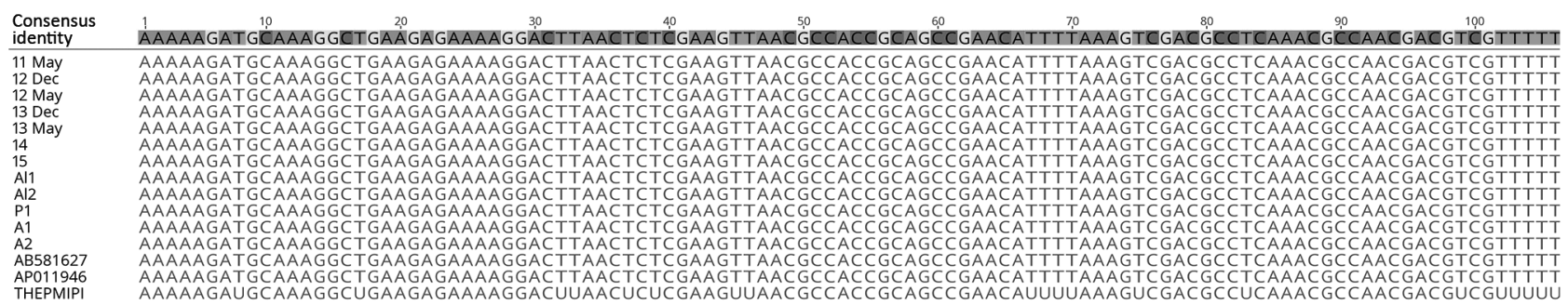

Figure 2. DNA sequences from 10 cattle from a farm in Albemarle County, Virginia, USA, infected with Theileria orientalis Ikeda genotype aligned with 3 GenBank sequences of $T$. orientalis genotype 2 for the major piroplasm surface protein. Alignment shows $100 \%$ consensus. Samples represent cattle from 6 different herds, and 2 samples were obtained at 2 time points. Nucleotides at the top indicate the consensus sequence. The GenBank sequence THEPMiPI is RNA with uracil substituted for thymine. A color version of this figure is available online (http://wwwnc.cdc.gov/EID/article/25/9/19-0088-F2.htm). 


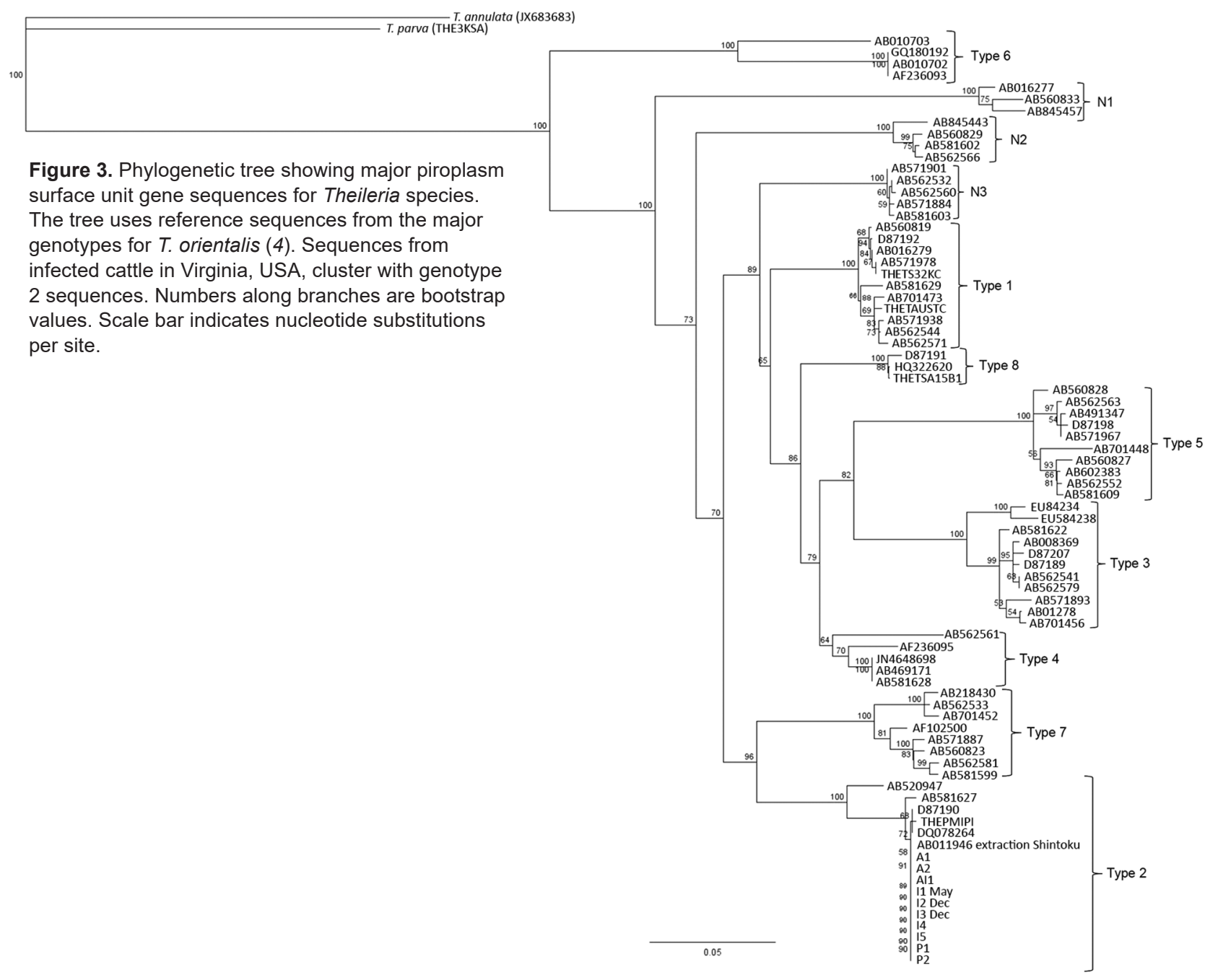

The presence of $T$. orientalis Ikeda/genotype 2 in Augusta County is of particular concern because this county has the second largest number of cattle in Virginia (23). Rockingham County, which produces most of the cattle in Virginia, is adjacent to Augusta and Albemarle Counties. Future studies are needed to determine the presence of $H$. longicornis ticks and $T$. orientalis Ikeda/genotype 2 in this area, but disease transmission to and within this area is of concern to cattle producers in Virginia. In countries in which $T$. orientalis Ikeda is established, the parasite contributes to economic losses through chronically ill animals. During 2010 in Australia, losses caused by T. orientalis Ikeda were estimated to be Aus $\approx \$ 20$ million $(5,10)$. In New Zealand, the cost of 1 outbreak on a large dairy farm was estimated to be $\approx 1$ million New Zealand dollars (5).

In this initial study of cattle in the United States, some of the animals that were positive for $T$. orientalis Ikeda by PCR remained positive 5 months later, suggesting a chronic state, although the animals that had initially exhibited clinical signs were no longer clinically ill during the spring and summer months. In New Zealand, disease caused by $T$. orientalis demonstrates seasonal pathogenicity, and anemia is more pronounced during autumn and winter months (12). Whether this trend is the case in Virginia will require future exploration. These cattle were also initially suspected to have anaplasmosis and had additional testing not been conducted, the detection of this pathogenic Theileria species might not have occurred. This study highlights the need for more surveillance and appropriate characterization of any parasites detected.

The source and timing of introduction of $T$. orientalis Ikeda/genotype 2 into the United States is unclear. One potential mechanism of introduction is through subclinically infected live cattle imported from disease-endemic regions. Approximately 200 live Wagyu cattle were imported from Japan during 1993-1997 before a ban on exports of Wagyu cattle by Japan in 1997 (24). Some of these same cattle were exported to Australia. It is possible that this or other 


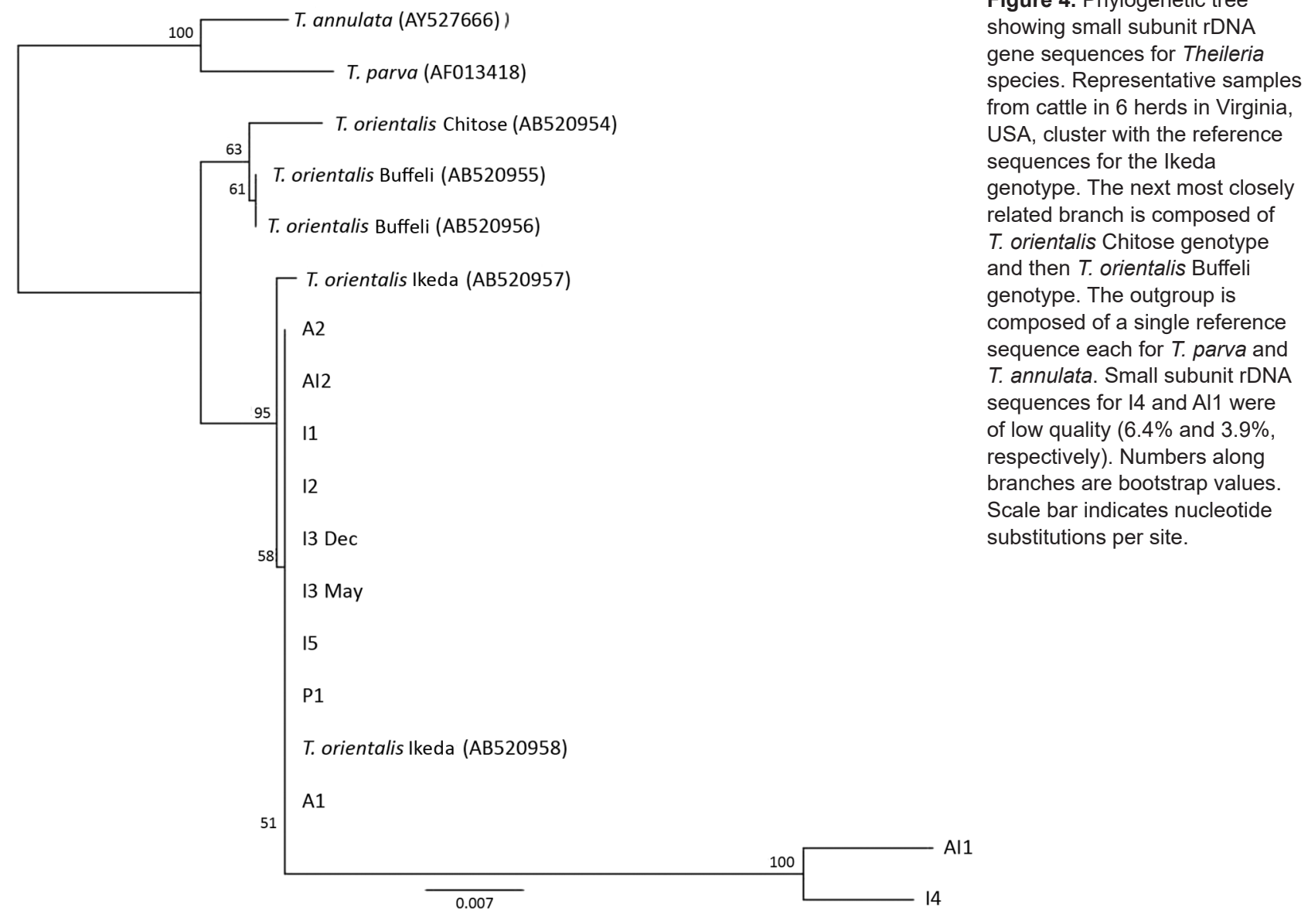

Figure 4. Phylogenetic tree showing small subunit rDNA gene sequences for Theileria species. Representative samples from cattle in 6 herds in Virginia, USA, cluster with the reference sequences for the Ikeda genotype. The next most closely and then $T$. orientalis Buffeli genotype. The outgroup is sequences for 14 and $\mathrm{Al} 1$ were of low quality $(6.4 \%$ and $3.9 \%$, respectively). Numbers along scale bar indicates nucleotide ubstitutions per site. similar live cattle trade introduced this genotype to the United States. Another possibility is transstadial transmission of $H$. longicornis ticks accidentally brought into the United States on other animals in shipments. Other genotypes of $T$. orientalis have been identified in the United States $(14,17,18)$, suggesting that there were previous introductions. Regardless, now that both the tick and the hemoprotozoan are established in this region, $H$. longicornis ticks will probably play a role in continued transmission of the parasite, and Theileriaassociated bovine infectious anemia will likely continue to occur within the area. A study modeling the most likely suitable habitats of the Asian longhorned tick throughout North America predicts that the tick will thrive along the eastern United States seaboard from Maine to North Carolina, along the western United States seaboard from Washington to northern California, and from northern Louisiana northward into Wisconsin and Ohio (25). With such a broad range, we predict $T$. orientalis Ikeda will become established in multiple states throughout the country.

\section{Acknowledgments}

We thank the farmers, producers, and veterinarians who have dedicated their time and effort to this study, often at their own inconvenience; in particular, Bruce Bowman, Brendan Martin, and Melinda McCall, who actively pursued this investigation.

\section{About the Author}

Dr. Oakes is a third-year resident in veterinary anatomic pathology and a second-year PhD student at the VirginiaMaryland College of Veterinary Medicine. Her research interests include infectious disease (particularly zoonoses), molecular diagnostics, and bioinformatics.

\section{References}

1. Watts JG, Playford MC, Hickey KL. Theileria orientalis: a review. N Z Vet J. 2016;64:3-9. https://doi.org/10.1080/00480169.2015.10 64792

2. Gubbels MJ, Hong Y, van der Weide M, Qi B, Nijman IJ, Guangyuan L, et al. Molecular characterisation of the Theileria buffeli/orientalis group. Int J Parasitol. 2000;30:943-52. https://doi.org/10.1016/S0020-7519(00)00074-6

3. Bogema DR, Micallef ML, Liu M, Padula MP, Djordjevic SP, Darling AE, et al. Analysis of Theileria orientalis draft genome sequences reveals potential species-level divergence of the Ikeda, Chitose and Buffeli genotypes. BMC Genomics. 2018;19:298. https://doi.org/10.1186/s12864-018-4701-2 
4. Sivakumar T, Hayashida K, Sugimoto C, Yokoyama N. Evolution and genetic diversity of Theileria. Infect Genet Evol. 2014;27: 250-63. https://doi.org/10.1016/j.meegid.2014.07.013

5. Yam J, Bogema DR, Jenkins C. Oriental theileriosis. Ticks and Tick-Borne Pathogens. 2018;1-31 [cited 2019 Jun 3]. https://www.intechopen.com/books/ticks-and-tick-bornepathogens/oriental-theileriosis

6. Heath A. Biology, ecology and distribution of the tick, Haemaphysalis longicornis Neumann (Acari: Ixodidae) in New Zealand. N Z Vet J. 2016;64:10-20. https://doi.org/10.1080/004801 69.2015.1035769

7. McFadden AM, Vink D, Pulford DJ, Lawrence K, Gias E, Heath AC, et al. Monitoring an epidemic of Theileria-associated bovine anaemia (Ikeda) in cattle herds in New Zealand. Prev Vet Med. 2016;125:31-7. https://doi.org/10.1016/ j.prevetmed.2015.11.005

8. Jenkins C. Bovine theileriosis: Molecular diagnosis and strain analyses. Meat and Livestock Australia Limited; 2017 Mar 16;1-111 [cited 2019 Jun 5]. https://www.mla.com.au/ research-and-development/search-rd-reports/final-report-details/ Animal-Health-and-Biosecurity/Bovine-theileriosis-Moleculardiagnosis-and-strain-analyses/3252

9. Theileria Working Group. Theileria veterinary handbook 2; 2015. Wellington (New Zealand): Ministry for Primary Industries; 2015. p. 1-32 [cited 2019 Jun 3]. https://www.mpi.govt. $\mathrm{nz} / \mathrm{dmsdocument} / 9518$-theileria-veterinary-handbook2-august-2015

10. Lawrence KE, Lawrence BL, Kickson RE, Hewitt CA, Gedye K, Fermin LM, et al. Associations between Theileria orientalis Ikeda type infection and the growth rates and haematocrit of suckled beef calves in the North Island of New Zealand. N Z Vet J. 2018;67:66-73. https://doi.org/10.1080/ 00480169.2018.1547227

11. Kamau J, de Vos AJ, Playford M, Salim B, Kinyanjui P, Sugimoto C. Emergence of new types of Theileria orientalis in Australian cattle and possible cause of theileriosis outbreaks. Parasit Vectors. 2011;4:22. https://doi.org/10.1186/ 1756-3305-4-22

12. Lawrence KE, Sanson RL, McFadden AMJ, Pulford DJ, Pomroy WE. The effect of month, farm type and latitude on the level of anaemia associated with Theileria orientalis Ikeda type infection in New Zealand cattle naturally infected at pasture. Res Vet Sci. 2018;117:233-8. https://doi.org/10.1016/ j.rvsc.2017.12.021

13. Rainey T, Occi JL, Robbins RG, Egizi A. Discovery of Haemaphysalis longicornis (Ixodida: Ixodidae) parasitizing a sheep in New Jersey, United States. J Med Entomol. 2018;55:757-9. https://doi.org/10.1093/jme/tjy006

14. Animal Plant and Health Inspection Service. Longhorned tick: information for livestock and pet owners. USDA. 2019 May [cited 2019 Jun 3]. https://www.aphis.usda.gov/publications/ animal_health/fs-longhorned-tick.pdf
15. Beard CB, Occi J, Bonilla DL, Egizi AM, Fonseca DM, Mertins JW, et al. Multistate infestation with the exotic disease-vector tick Haemaphysalis longicornis - United States, August 2017September 2018. MMWR Morb Mortal Wkly Rep. 2018;67: 1310-3. https://doi.org/10.15585/mmwr.mm6747a3

16. Chen Z, Yang X, Bu F, Yang X, Liu J. Morphological, biological and molecular characteristics of bisexual and parthenogenetic Haemaphysalis longicornis. Vet Parasitol. 2012;189:344-52. https://doi.org/10.1016/j.vetpar.2012.04.021

17. Cossio-Bayugar R, Pillars R, Schlater J, Holman PJ. Theileria buffeli infection of a Michigan cow confirmed by small subunit ribosomal RNA gene analysis. Vet Parasitol. 2002;105:105-10. https://doi.org/10.1016/S0304-4017(02)00003-1

18. Stockham SL, Kjemtrup AM, Conrad PA, Schmidt DA, Scott MA, Robinson TW, et al. Theileriosis in a Missouri beef herd caused by Theileria buffeli: case report, herd investigation, ultrastructure, phylogenetic analysis, and experimental transmission. Vet Pathol. 2000;37:11-21. https://doi.org/10.1354/vp.37-1-11

19. Kubota S, Sugimoto C, Kakuda T, Onuma M. Analysis of immunodominant piroplasm surface antigen alleles in mixed populations of Theileria sergenti and T. buffeli. Int J Parasitol. 1996;26:741-7. https://doi.org/10.1016/0020-7519(96)00047-1

20. Kamau J, Salim B, Yokoyama N, Kinyanjui P, Sugimoto C. Rapid discrimination and quantification of Theileria orientalis types using ribosomal DNA internal transcribed spacers. Infect Genet Evol. 2011;11:407-14. https://doi.org/10.1016/j.meegid.2010.11.016

21. Carelli G, Decaro N, Lorusso A, Elia G, Lorusso E, Mari V, et al. Detection and quantification of Anaplasma marginale DNA in blood samples of cattle by real-time PCR. Vet Microbiol. 2007;124:107-14. https://doi.org/10.1016/j.vetmic.2007.03.022

22. Kakuda T, Shiki M, Kubota S, Sugimoto C, Brown WC, Kosum C, et al. Phylogeny of benign Theileria species from cattle in Thailand, China and the U.S.A. based on the major piroplasm surface protein and small subunit ribosomal RNA genes. Int J Parasitol. 1998;28:1261-7. https://doi.org/10.1016/ S0020-7519(98)00113-1

23. USDA National Agriculture Statistics Service. Cattle county estimates_January 1, 2017 [cited 2019 Mar 24]. https://www.nass.usda.gov/Statistics_by_State/Virginia/ Publications/County_Estimates/Cattle17_VA.pdf

24. Wagyu International. History of Wagyu exports from Japan; 2013 [cited 2010 Mar 25]. http://www.wagyuinternational.com/ global_USA.php

25. Rochlin I. Modeling the Asian longhorned tick (Acari: Ixodidae) suitable habitat in North America. J Med Entomol. 2019;56:38491. https://doi.org/10.1093/jme/tjy210

Address for correspondence: Kevin K. Lahmers, Department of Biomedical Sciences and Pathobiology, Virginia-Maryland College of Veterinary Medicine, 205 Duck Pond Dr, Blacksburg, VA 24060, USA; email: klahmers@vt.edu 